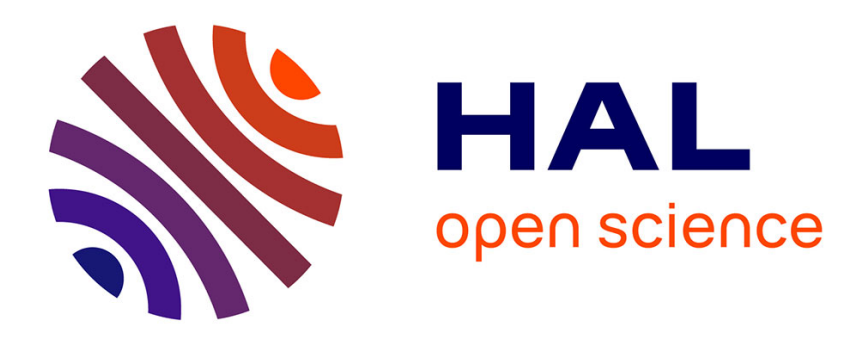

\title{
Varron et la constitution des paradigmes flexionnels du latin
}

\author{
Alessandro Garcea
}

\section{To cite this version:}

Alessandro Garcea. Varron et la constitution des paradigmes flexionnels du latin. Histoire Epistémologie Langage, 2008, 30 (1), pp.75-90. halshs-00332764

\section{HAL Id: halshs-00332764 https://shs.hal.science/halshs-00332764}

Submitted on 26 Jun 2015

HAL is a multi-disciplinary open access archive for the deposit and dissemination of scientific research documents, whether they are published or not. The documents may come from teaching and research institutions in France or abroad, or from public or private research centers.
L'archive ouverte pluridisciplinaire HAL, est destinée au dépôt et à la diffusion de documents scientifiques de niveau recherche, publiés ou non, émanant des établissements d'enseignement et de recherche français ou étrangers, des laboratoires publics ou privés. 


\title{
VARRON ET LA CONSTITUTION DES PARADIGMES FLEXIONNELS DU LATIN*
}

\author{
Alessandro Garcea
}

Université de Toulouse 2 - CNRS, UMR 7597

\begin{abstract}
RÉSUMÉ : Au livre X du De lingua Latina, Varron esquisse un modèle mathématique de flexion nominale du latin: nous nous proposons de mettre en évidence les rapports possibles entre ce célèbre passage et la littérature mathématique antique, après avoir évoqué la fonction que celle-ci remplissait dans la culture romaine de l'époque républicaine. Nous nous intéresserons également à la postérité du modèle varronien - et notamment au terme formula qui le désigne - dans les artes grammaticae d'époque tardive.
\end{abstract}

MOTS-CLÉS: Varron, Nicomaque de Gérase, grammatici Latini, flexion, formula, mathématiques (grammaire et -), Antiquité latine.

\begin{abstract}
In book $\mathrm{X}$ of his De Lingua Latina, Varro conceived a mathematical model of Latin inflection. After taking into account the function of mathematics in Roman republican culture, we will show the relationship between this famous passage and the ancient mathematical literature. We will also study the indirect influence of the Varronian model - especially through the occurrences of the technical word formula that defines it - on late Latin grammarians.

KEYWORDS : Varro, Nicomachus of Gerasa, grammatici Latini, inflection, formula, mathematics (grammar and -), Latin Antiquity
\end{abstract}

\section{INTRODUCTION : LE LOCVS HERMÉNEUTIQUE}

À LA FIN de la République romaine, l'intérêt général pour les aspects théoriques des mathématiques et de la géométrie s'avère très faible. L'attitude traditionnelle des Romains consistait à limiter l'étude des savoirs aux aspects pragmatiques et utilitaires, ainsi que le rappelle le personnage de Scipion au livre V du De republica de Cicéron : «Comme l'intendant d'une ferme connaît la nature du sol, l'intendant de la ville sait lire et écrire : tous deux néanmoins visent l'utilité pratique et non le plaisir intellectuel » (§ 5). Presque dix années plus tard, quand il comparera le développement des activités culturelles en Grèce et dans sa patrie, au tout début du livre I des Tusculanes, Cicéron déplorera cette situation : « Les Grecs tenaient en très grand honneur la géométrie : c'est pourquoi rien ne fut plus glorieux que les mathématiques ; mais nous, nous avons limité cette science aux pratiques de la mesure et du calcul » $(\S 5)$. La considération pour la manière dont ces disciplines s'étaient constituées, pour leurs subdivisions et pour leurs agencements internes, était pourtant loin d'occuper les seuls spécialistes.

* Nous remercions chaleureusement Fabio Acerbi, Jean Lallot et Valeria Lomanto pour leurs remarques très utiles sur ce texte. Les traductions des auteurs anciens sont les nôtres, sauf celles de Nicomaque, empruntées à Bertier 1978, parfois remaniées. 
Dans toute œuvre de systématisation d'un savoir - dont l'unité réside non simplement dans l'accumulation lente et progressive de contenus, mais surtout dans la forme rigoureuse que ceux-ci prennent quand ils s'inscrivent dans un cadre théorique solide -, la géométrie, l'astronomie ou la musique pouvaient montrer la voie à ceux qui cherchaient une méthode «qui cimentât une matière fragmentée et désagrégée, et qui la réunît dans un système [ratio] », ainsi que l'affirme le personnage de Crassus à propos du droit au livre I du De oratore cicéronien $(\S 188)^{1}$.

Disciple du stö̈cien Diodote qui, tout en étant aveugle, enseignait la dialectique et la géométrie ${ }^{2}$, Cicéron fut également très influencé par la pensée d'un autre stoïcien, Posidonius, dont on connaît la célèbre ambassade à Rome en 86, à l'occasion de laquelle il fit un séjour prolongé dans la Ville et prit contact avec les plus illustres hommes politiques de l'époque, entre autres avec Pompée. L'importance des concepts mathématiques dans le cadre de la réflexion générale de ce philosophe ressort notamment de sa définition de l'âme où, à partir d'une relecture du Timée (35A), il combine des éléments géométriques, arithmétiques et harmoniques qui lui permettent de retrouver un «pedigree » pythagoricien dans la théorie platonicienne de l'âme' .

Il ne faut donc pas s'étonner si, sans être des mathématiciens, les lettrés romains s'intéressaient à cette science, ni si un polygraphe comme Varron a pu écrire des monographies aux titres éloquents (seule chose qui nous en reste, hélas !) comme De mensuris, De geometria (peut-être s'agit-il d'un même ouvrage) et De principiis numerorum. Son intérêt pour la mystique des nombres pythagoricienne, doctrine à laquelle il se réfère explicitement ${ }^{4}$, permettrait d'expliquer la présence de structures fondées sur les chiffres trois, quatre, sept et neuf dans ses œuvres, notamment les taxinomies quadripartites du De lingua Latina ${ }^{5}$.

\section{LE DE LINGVA LATINA DE VARRON}

Nos remarques liminaires sur le locus herméneutique où se situe notre analyse conduisent à considérer avec quelque réserve l'étonnement de chercheurs comme Jean Collart, pour qui «On trouve même, à propos de la similitude des noms, une curieuse théorie arithmétique de l'analogie [...] théorie plus étrange que convaincante » (1954, p. 155 et n. 1), ou comme Daniel J. Taylor, pour qui « For a Roman [...] to apply abstract models to specific data like grammatical forms is, if not curious, at least unexpected $»(1996$, p. 148).

Ces deux avis émanent de la lecture d'un passage du livre X du De lingua Latina de Varron où, après avoir abordé la question générale de la ressemblance et de la différence (quid sit simile et dissimile) et une fois définie la notion de rapport

1 Sur cet effort de rationalisation du savoir, $c f$. Moatti 1997, p. 39-40.

2 Cf. Cicéron Tusc. 5,113 ; sur Diodote, cf., en dernier lieu, Ducos 1994.

$3 C f$. Posidonius frg. 141a Edelstein \& Kidd = 391a Theiler tèn psukhèn idéan ê̂nai tô̂ pántēi diastatoû kat' arithmòn sunestōsan harmonían periékhonta « L'âme est la forme de ce qui se répand en tout lieu, conçue en conformité avec un nombre qui embrasse l'harmonie » (chez Plutarque an. procr. 1023b) ainsi que l'important article de Ju 2006.

4 Les données principales sont résumées par Collart 1954, p. 38.

$5 C f$., en dernier lieu, Piras 1998, p. 41-56, ainsi que l'article de Blank dans ce volume. 
(quid sit ratio) dans une section qui n'a pas été conservée, l'auteur s'intéresse $\mathrm{au}$ « rapport proportionnel » (quid sit ratio pro portione), interprété comme un principe général d'analogie ${ }^{6}$ :

Cela arrive entre des objets différents, par exemple dans les nombres, si on compare deux avec un, vingt avec dix. En effet, le rapport de deux à un est le même que celui de vingt à dix. [...] Les poètes utilisent souvent ce type de rapport dans leurs comparaisons et les géomètres s'en servent de manière très pénétrante ${ }^{7}$.

La référence aux poètes ne saurait échapper au lecteur du chapitre XXI de la Poétique d'Aristote, où figure l'explication de la métaphore par analogie, celle qui permet de dire, dans des conditions contextuelles appropriées, «bouclier» pour « coupe » ou « coupe » pour « bouclier », selon la proportion :

\section{DIONYSOS : COUPE :: ARÈS : BOUCLIER}

ou encore de former des syntagmes comme «bouclier de Dionysos » ou « coupe d'Arès », avec l'incorporation de l'élément contextuel pertinent dans la métaphore elle-même ${ }^{8}$.

L'allusion à la géométrie en tant que domaine d'application de la théorie des proportions n'est que le reflet de l'idée, commune chez les Anciens, selon laquelle les grandeurs géométriques et les nombres constituent deux objets d'étude autonomes au sein des mathématiques. Ainsi, dans ses Éléments, Euclide expose, d'abord, au livre $\mathrm{V}$ une théorie des proportions valable pour les grandeurs géométriques générales visualisées par des segments ${ }^{9}$, puis, au livre VII, après avoir donné la définition des objets numériques, développe une théorie des proportions autonome ${ }^{10}$.

6 La tentative, par Taylor (1996a, comm. ad § 42 ; 1996b, p. 97-100), de changer profondément l'ordre du texte transmis par les manuscrits, pour présenter une séquence de quatre types d'analogie dont la difficulté serait croissante (ling. 10,42 + 50b [section supprimée dans l'édition Mette 1952], suivis des § 45-50a. 43-44. 51), nous paraît complètement arbitraire et fondamentalement immotivée. Sur l'analogie dans des domaines autres que la grammaire, $c f$. Siebenborn 1976, p. 56-62; sur les traductions latines d'analogia, ainsi que sur les réinterprétations qu'elles présupposent : Schironi 2007.

7 Cf. Varron ling. 10,41-42 haec fiunt in dissimilibus rebus, ut in numeris si contuleris cum uno duo, sic cum decem viginti: nam <quam> rationem duo ad unum habent, eandem habent uiginti ad decem [...]. 42. hoc poetae genere in similitudinibus utuntur multum, hoc acutissime geometrae.

8 Comme le remarquent très justement Dupont-Roc \& Lallot (1981, p. 347), « La métaphore n'opère que sur trois termes, puisque aussi bien elle consiste dans le remplacement du quatrième. On conçoit alors que ce dernier puisse manquer : au lieu de remplacer un nom existant [...] la métaphore reviendra ici à remplir la "case vide" d'un nom inexistant ». C'est le cas de la proportion grain : semer :: feux du soleil : $\varnothing$, qui autorise l'emploi métaphorique du verbe « semer» (speírein) dans le cas de la lumière du soleil, pour laquelle, en grec, il n'y a pas de terme spécifique indiquant la même action de «jeter au loin » (aphiénai) qui a été lexicalement spécifiée pour les grains.

9 Acerbi (2007, p. 340) remarque que «Il segmento, oltre a rappresentare di per sé una grandezza geometrica piuttosto tipica, va letto molto aristotelicamente qua substrato minimale, spogliato di ulteriori caratteristiche accidentali, cui siano applicabili le operazioni tipiche agenti sulle grandezze [...]. È quindi tutto sommato legittimo vedere nei segmenti delle grandezze astratte e non solo la rappresentazione ottimale di grandezze astratte ».

10 Acerbi (2007, p. 367) observe que « La teoria delle proporzioni esposta nei libri aritmetici 
Une fois précisée la nature du « rapport proportionnel » dans les autres champs du savoir, Varron rappelle que, suivant l'enseignement d'Aristarque, cinquième directeur en titre de la bibliothèque d'Alexandrie, les grammairiens alexandrins appliquent ce type de relation à la langue :

$[\ldots]$ comme quand ils affirment qu' amorem $_{\text {acc. }}$ : amori $_{\text {dat. }}$ [amour] et dolorem $_{\text {acc. }}$ : dolori $_{\text {[douleur] sont semblables en proportion, quoiqu'ils voient bien }}$ qu'amorem est différent d'amori, ainsi dolorem, de dolori, puisqu'il s'agit de cas différents ; mais ils l'affirment du fait qu'il s'agit de couples semblables ${ }^{11}$.

Si donc, de son propre aveu, Varron n'est pas le premier à appliquer la proportion à quatre termes à la langue ${ }^{12}$, il émet probablement un avis original ${ }^{13}$ quand il propose de créer une matrice (formula), où des rapports identiques se forment aussi bien « sur la verticale », entre des formes casuelles distinctes, que « sur l'horizontale », entre des formes dont le genre est différent :

Une proportion est parfois constituée de deux rapports imbriqués, tels que l'un constituerait une verticale et l'autre la perpendiculaire. Voici qui éclairera mon propos. Soient des nombres ainsi disposés : sur la première ligne, un, deux, quatre ; sur la deuxième, dix, vingt, quarante ; sur la troisième, cent, deux cents, quatre cents. Dans ce tableau numérique seront présents les deux rapports dont j'ai parlé, et qui forment des types différents d'analogie : l'un est le rapport du double sur l'horizontale, à savoir d'un à deux, comme de deux à quatre ; l'autre est le rapport du décuple sur la verticale, à savoir d'un à dix, comme de dix à cent. 44. De la même façon, dans la flexion des noms se développent deux directions quand ceux-ci passent du nominatif aux cas obliques d'une part, et du nominatif à un autre nominatif d'autre part. Ils forment ainsi, de manière semblable, un tableau qui a sur la première ligne $a l b u s_{\text {nom. }} a_{l b o}{ }_{\text {dat. }} a l b i_{\text {gén. }}$. blanc, $\mathrm{m}$.] ; sur la deuxième, alba $a_{\text {nom }}$ albae $_{\text {dat }}$ albae $_{\text {gén }}$ [blanche, f.] ; sur la troisième, album $_{\text {nom. }}$ albo $_{\text {dat. }}$ albi $_{\text {gén. }}$ [blanc, n.]. C'est ainsî́ qu'on en tire, par les déclinaisons disposées horizontalement, des analogies du type Albius Atrius, Albio Atrio,

è indipendente da quella del libro $\mathrm{V}$ (d'altronde, i numeri non sono grandezze). [...] La teoria esposta nel libro VII non ha neanche l'ambizione di ridimostrare tutti i risultati validi per le grandezze [...]. L'elaborazione è dunque parziale, mirante a dimostrare i risultati strettamente necessari al prosieguo della trattazione ».

11 Cf. Varron ling. 10,42 ut cum dicuntur pro portione similia esse amorem amori, dolorem dolori, cum ita dissimile $[\mathrm{m}]$ esse uideant amorem [et dolorem] <amori>, quod est alio casu, item dolorem dolori, sed dicunt, quod ab similibus. Ce passage doit être mis en rapport avec le peu qui reste de la section sur la ratio, où Varron explique que l'on peut établir un rapport identique entre des mots différents, par exemple amor : amori :: dolor : dolori, mais qu'il n'est pas correct d'associer dans un rapport de ce type des formes issues du même lexème (materia), par exemple amor: amoris :: amores : amorum (ling. 10,36).

12 La plus ancienne méthode d'application des critères analogiques en grammaire consiste en la comparaison entre une forme douteuse et une forme sûre afin d'identifier le schéma flexionnel de celle-ci ou une catégorie grammaticale (cf. Siebenborn 1976, p. 63-64 ; Callanan 1987, p. 115-116). Cette comparaison très empirique fut remplacée par la proportion à quatre termes, deux lemmes et deux formes fléchies ( $c f$. Siebenborn 1976, p. 64-66), déjà employée par Aristarque ( $c f$. Erbse 1980, p. 237-238) et dont Varron donne plusieurs exemples, moins en vue de déduire des trois éléments connus le quatrième douteux que pour démontrer la régularité du système morphologique ( $c f$. ., en dernier lieu, Duso 2006).

13 Ainsi Swiggers (1997, p. 77) : «Varron est le premier à observer la double directionnalité de la proportion dans les formes fléchies : la directionnalité proportionnelle qui permet d'identifier des ensembles de formes [...], et la directionnalité proportionnelle qui permet de préciser la valeur de séries de formes (à l'égard d'autres) ». 
ce qui constituera une partie de la progression du double ; par les déclinaisons disposées verticalement, des analogies du type Albius Atrius, Albia Atria, ce qui constituera bien entendu une partie de la progression du décuple dont j'ai parlé ci-dessus ${ }^{14}$.

Ce passage propose d'identifier l'image du tableau numérique avec la structure du paradigme flexionnel d'un nom ${ }^{15}$ :

\begin{tabular}{|c|c|c|c|}
\hline & \multicolumn{3}{|c|}{ RAPPORTS DU DOUBLE } \\
\hline \multirow{3}{*}{ 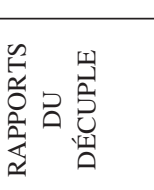 } & 1 & 2 & 4 \\
\hline & 10 & 20 & 40 \\
\hline & 100 & 200 & 400 \\
\hline & NOMINATIF & DATIF & GÉNITIF \\
\hline MASCULIN & albus & albo & albi \\
\hline FÉMININ & alba & albae & albae \\
\hline NEUTRE & album & albo & albi \\
\hline
\end{tabular}

Les expressions techniques: formula pour le tableau numérique ${ }^{16}$, rationes

$14 C f$. Varron ling. 10,43-44 nonnunquam rationes habet implicatas duas, ut sit una derecta, altera transuersa. quod dico, apertius sic fiet. esto sic expositos esse numeros, ut in primo uersu sit unum duo quattuor, in secundo decem uiginti quadraginta, in tertio centum ducenti quadringenti. in hac formula numerorum duo inerunt quos dixi logoe, qui diuersas faciant analogias: unus duplex qui est in obliquis uersibus, quod est ut unus ad duo, sic duo ad quattuor; alter decemplex in directis ordinibus, quod est ut unum ad decem, sic decem ad centum. 44. similiter in uerborum declinationibus est biuium, quod et ab recto casu $<$ declinantur in obliquos et ab recto casu $>$ in rectu $<m>$, ita ut formulam similiter efficiant, quod sit primo uersu hic albus, huic albo, huius albi, secundo $h<a>e c$ alba, huic albae, huius albae, tertio hoc album, huic albo, huius albi. itaque fiunt per obliquas declinationes ex his analogiae hoc genus Albius Atrius, Albio Atrio, per directas declinationes Albius Atrius, Albia Atria: quae scilicet erit particula ex illa †uicenaria [Atria quae scilicet] centenaria formula analogiarum, de qua supra dixi. La fin du $\$ 44$ n'est pas intelligible : la plupart des éditeurs intègrent binaria après la série Albius Atrius, Albio Atrio et gardent centenaria pour la série Albius Atrius, Albia Atria, tout en supprimant le reste du texte qui ne fait pas sens. Goetz \& Schoell (1910, p. 291 note ad 183,11 sqq.), en revanche, affirment : « Neque uicenaria neque centenaria cum ferri possit, melius fortasse utrumque secludas tamquam inepte additum quam mutationes cumules : certe nihil desideratur si legas : ex illa formula analogiarum de qua supra dixi ».

15 Pour Law (2003, p. 48-49), les formes situées sur l'axe vertical «Differ only in gender, even as in the numerical grid 1,10 and 100 differ only in order of magnitude »; les formes disposées horizontalement « Are qualitatively different in that they cannot function in the same way in a sentence. Analogously, 1, 2 and 4 are qualitatively distinct in Pythagorean arithmetic, which focussed on the unique properties of "unity", "duality", "fourness" and so on ».

16 Conso (1990, p. 1591) propose d'interpréter le mot latin formula comme «"texte modèle" (juridique, mathématique ou administratif) [...] de portée générale, qui énonce une règle, impose une obligation ou notifie une volonté ; qui s'applique, moyennant certaines adaptations, convenues par avance, à tous les cas particuliers ». Pour ce qui est de la valeur 
implicatae pour les rapports «imbriqués», derecta et transuersa pour les dispositions verticale et horizontale des proportions, esto sic expositos esse numeros pour la collocation des nombres dans le tableau, jusqu'à l'hellénisme logoe pour les deux proportions, font songer à un traité mathématique grec comme source du paragraphe. Même en l'absence de parallèles précis, du fait de la disparition des textes écrits entre les $\mathrm{II}^{\mathrm{e}}$ et $\mathrm{I}^{\mathrm{er}}$ siècles av. J.-C., il nous paraît important de remarquer que le recours aux tableaux arithmétiques s' insère parfaitement dans une tradition arithmétique particulière, représentée, entre les $\mathrm{I}^{\mathrm{er}}$ et $\mathrm{II}^{\mathrm{e}}$ siècles apr. J.-C., par Nicomaque de Gérase ${ }^{17}$. Ainsi, au livre I de son Introduction arithmétique, ce dernier introduit la «table de Pythagore » par la phrase éstō dè tò diágramma toioûton « Soit le diagramme de cette sorte » $(\$ 19,9)$, et décrit la manière dont les nombres sont disposés comme suit :

Disposons sur la première ligne le nombre naturel à partir de l'unité, et ensuite successivement, parmi les espèces du multiple, les nombres requis ${ }^{18}$.

Puis, après avoir montré les rapports du double et du triple, Nicomaque prend en considération ceux du quadruple, désignés par la ligne numérique qui, dans la table de Pythagore, a son origine dans le seize et qui va jusqu'au quarante, aussi bien sur l'horizontale que sur la verticale. Il remarque que les différences entre ces nombres sont, dans l'ordre, trois, six, neuf, douze, et que la quotité de chaque multiple augmente à chaque fois de trois unités. Ainsi :

Celles-ci [sc. les quotités] sont retrouvées dans le tissu du diagramme, disposées en ordre au-dessus des mêmes quadruples, et cette analogie se poursuit jusqu'au bout pour les espèces suivantes de multiple ${ }^{19}$.

Comme pour Nicomaque, ce tableau à double entrée $(\text { formula-diágramma })^{20}$ devait pour Varron servir moins à faire des calculs (voire à trouver des formes lexicales),

de «tableau de référence », outre les occurrences varroniennes, il existe des formulae où est enregistré, en regard du nom des individus ou des peuples entrés dans la sphère d'influence de Rome, le montant de leurs droits ou de leurs obligations (p. 1593-1604) : les formulae togatorum, sociorum, amicorum.

17 L'existence d'une tradition qui se manifestait dans la production d'artes arithmeticae est prouvée par les parallélismes entre l'œuvre de Nicomaque et celles, indépendantes, de Philon d'Alexandrie et de Théon de Smyrne. Pour un aperçu, $c f$. D'Ooge, Robbins \& Karpinski 1926, p. 16-45.

$18 C f$. Nicomaque intr. 1,19,10 ekkeísthō en mèn tōi prốtōi stíkhōi ho apò monádos phusikòs arithmós, êta hexês hoi keleusthéntes tōn toû pollaplasiou eidōn. Dans leur très utile glossaire de Nicomaque, Robbins \& Karpinski (1926, p. 297), attribuent à ékkeimai le sens de «to be set forth as terms in a problem or operation » et, notamment dans le passage in question, «to be placed, located». Je remercie F. Acerbi pour avoir attiré mon attention sur ce verbe.

$19 C f$. Nicomaque intr. 1,19,13 kaì haûtai en tōi toû diagrámmatos húphei pephốrantai táxei ekkeímenai hupèr autoùs toùs tetraplasíous kaì epì tōn akoloúthōn tô̂ pollaplasíou eidōn tò análogon mékhri pantòs prokhōrê̂. En d'autres termes : pour les nombres 1234 5, les quadruples sont : 481216 20, les différences sont: 36912 13. Pour le terme húphos, $c f$. Robbins \& Karpinski (1926, p. 309) : «A web, hence, structure, disposition of a table ».

20 Avec descriptio et forma, le mot formula sera employé par Boèce, dans son De institutione arithmetica, pour traduire diágramma de Nicomaque : $c f$. par exemple le titre ratio atque expositio digestae formulae $(1,27)$, qui se réfère à la table de Pythagore illustrée en 1,26,2. 
finalité propre de l'abaque ${ }^{21}$, qu'à corroborer des considérations théoriques sur la proportionnalité des nombres (voire des formes lexicales) qui y étaient disposés (ekkeísthai-exponi) $)^{22}$. C'est ainsi que, presque insensiblement, Varron peut faire la transition d'un domaine à l'autre. Après avoir appelé les deux proportions, l'une derecta, l'autre transuersa ( $\$ 43)$, il passe au binôme synonymique directus - obliquus, qui vaut aussi bien pour les nombres que pour les cas : la disposition de ces derniers dans le tableau, en effet, produit des directae et obliquae declinationes (§ 44), correspondant aux cas (casus) appelés respectivement rectus et obliqui.

Déjà quelques paragraphes plus haut, dans le cadre de la section sur les critères de ressemblance entre les formes qui relèvent de la catégorie nominale (nominatus), Varron avait utilisé l'image du tableau où se croisent deux dimensions, les ordines derecti et les transuersi, pour illustrer la flexion nominale. N'ayant toutefois pas encore introduit le concept mathématique de proportion, il avait comparé ce schéma non à une matrice numérique mais au ludus latrunculorum, un jeu assimilable au moderne backgammon. Ce jeu se jouait sur une sorte d'échiquier (tabula lusoria) rectangulaire, où se situaient trente-six carrés sur trois lignes de douze, lignes qui étaient coupées en deux moitiés identiques, de manière à former deux champs distincts de dix-huit carrés chacun, à savoir de trois lignes de six carrés chacune dans chaque moitié du rectangle ${ }^{23}$ :

Deux séries de droites, les unes horizontales, les autres verticales, sont tracées en conformité avec cette source quadripartite ${ }^{24}$, comme il est d'usage de le faire pour l'échiquier où l'on joue avec des pièces. Les droites horizontales correspondent aux cas obliques, qui sont fléchis à partir du nominatif, par exemple albus $_{\text {nom. }}$ albi $i_{\text {gén. }}$ albo $o_{\text {dat. }}$ [blanc]; les droites verticales correspondent aux formes au nominatif tirées d'un autre nominatif, par exemple $a l b u s_{\mathrm{m}} a l b a_{\mathrm{f}}$ album $_{\mathrm{n} .}$. L'une comme l'autre, ces séries ont six segments : les segments des droites horizontales s'appellent cas, ceux des droites verticales s'appellent genres, et leur intersection donne une forme ${ }^{25}$.

La définition de forma comme le résultat de l'intersection des variations casuelles et des variations de genre correspond à un usage technique souvent attesté dans les livres morphologiques du De lingua Latina ${ }^{26}$. Dans ce passage, il est explicité

21 L'abaque romain est constitué d'un rectangle de bronze, pourvu de colonne décimales divisées en deux, qui servent à comptabiliser des as à l'aide de boutons glissés vers le haut ou vers le bas (hormis les colonnes dernière et avant-dernière de droite, réservées à l'once et à ses fractions). $C f$. Fellmann 1983.

22 Cf. Siebenborn (1976, p. 65) : «So ist die viergliedrige Proportion bei Varro nicht Erschließungsprinzip, sondern Instrument zur Beschreibung und Demonstration des sprachlichen Phänomens der Flexionsgleichheit».

23 L'étude de Barresi (1998-2000) sur ce type d'objet démontre de manière convaincante que c'est à ce modèle que Varron fait allusion.

24 Varron vient d'illustrer les critères de l'analogie nominale, qui pour lui se réduisent à genre (genus), espèce (species), cas (casus), terminaison (exitus). Cf. Garcea 2007, p. 343-344.

$25 C f$. Varron ling. 10,22 ad hunc quadruplicem fontem ordines deriguntur bini, uni transuersi, alteri derecti, ut in tabula solet in qua latrunculis ludunt. transuersi sunt qui ab recto casu obliqui declinantur, ut albus albi albo; derecti sunt qui ab recto casu in rectos declinantur, ut albus alba album; utrique sunt partibus senis. transuersorum ordinum partes appellantur casus, derectorum genera, utrisque inter se implicatis forma $[\mathrm{m}]$.

26 Le mot forma désigne souvent les variations auxquelles se prête un lexème pour pouvoir jouer en contexte un certain rôle sémantico-syntaxique : cf. ling. 8,9 et 9,21 (pour toutes 
par une sorte de métaphore implicite du tissage, telle qu'elle apparaît aussi dans le texte de Nicomaque cité plus haut en lien avec un tableau de nombres : en effet, aussi bien les lignes de la tabula lusoria (§ 22) que celles de la formula (§ 43) sont appelées ordines, « rangées », mot dérivé d'ordiri, litt. « ourdir une trame » ${ }^{27}$.

Suivant le modèle habituel d'une tabula lusoria, comme celle de Perges ${ }^{28}$, le contenu du $§ 22$ peut dont être visualisé comme suit :

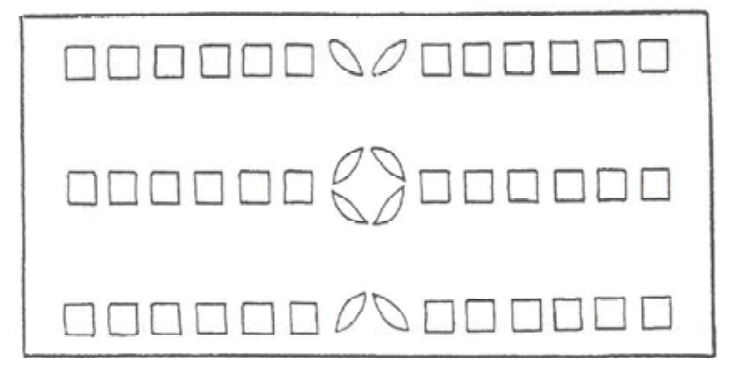

\begin{tabular}{|c|c|c|c|c|c|c||c|c|c|c|c|c|}
\hline & NOM. & GÉN. & DAT. & ACC. & VOC. & ABL. & NOM. & GÉN. & DAT. & ACC. & VOC. & ABL. \\
\hline & albus & albi & albo & album & albe & albo & albi & alborum & albis & albos & albi & albis \\
\hline F. & alba & albae & albae & albam & alba & alba & albae & albarum & albis & albas & albae & albis \\
\hline N. & album & albi & albo & album & albe & albo & alba & alborum & albis & alba & alba & albis \\
\hline
\end{tabular}

Le recours aux proportions permet de rendre compte des dynamiques flexionnelles implicites dans ce schéma. Ainsi, après avoir présenté la matrice formée par deux rapports imbriqués, Varron fait la distinction entre proportions discontinues (genus deiunctum: par exemple $1: 2:: 10: 20$ ) et proportions continues (genus coniunctum : par exemple $1: 2:: 2: 4)$, qu'il compare aux deux tétracordes formés par les sept cordes de la lyre et à la symptomatologie du quatrième jour dans les maladies à cycle hebdomadaire (\$ 45-46). Ces dernières remarques devaient être assez fréquentes dans les études arithmologiques anciennes. Le chiffre sept, en particulier, avait inspiré à Varron l'œuvre Hebdomades uel de imaginibus, un recueil de sept-cent portraits d'hommes illustres assortis de courtes descriptions en prose et en vers, dont le premier livre aurait constitué une étude complète des vertus du sept ${ }^{29}$. Qu'il s'agisse de matériaux provenant d'Antiochus d'Ascalon ${ }^{30}$ ou d'un prétendu commentaire de Posidonius au Timée de Platon ${ }^{31}$, les exemples

les catégories de mots variables) ; 8,33. 47 ; 10,56. 70 (pour les noms) ; 9,101. 109 (pour les verbes).

27 Sur ordo dans le livre X du De lingua Latina, cf. Taylor 1978.

28 L'image est tirée de Bean 1968, p. 55, fig. 7, détail.

29 Cf. Aulu-Gelle 3,10,1 «Varron dans le premier livre de ses Hebdomades ou Portraits, énumère de nombreuses et diverses propriétés et facultés du nombre sept, que les Grecs appellent hebdomas ».

30 Cf. Grilli 1979.

$31 C f$. Roscher 1906, mais l'existence elle-même de cet ouvrage est controversée : $c f$. Edelstein \& Kidd 1988, 2.1, comm. frg. 85, p. 337-343. Pour les références à Platon par Posidonius, $c f$., en dernier lieu, Ju (à paraître). 
cités dans ce texte, selon la reconstitution la plus probable ${ }^{32}$, devaient contenir des renseignements précis sur le rôle du sept dans les proportions ${ }^{33}$, dans les maladies ${ }^{34}$ et dans la musique ${ }^{35}$.

Pour ce qui est des deux types de proportions, au livre II de son Introduction arithmétique, après les avoir appelés sunēmménē analogía $(\$ 21,5)$ et diezeugménē analogía $(\S 21,6)^{36}$, Nicomaque observe que leur origine remonte très loin dans le temps :

Parmi les proportions donc, il y a les premières, reconnues par tous les anciens, Pythagore, Platon et Aristote, les trois fondamentales, l'arithmétique, la géométrique, l'harmonique, et les trois autres qui leur sont contraires, qui n'ont pas reçu de dénominations particulières, mais sont généralement appelées quatrième, cinquième, sixième médiétés ; après elles, les auteurs plus récents en trouvent quatre autres encore, en qui ils achèvent le dixième nombre, qui de l'avis des Pythagoriciens est le plus parfait. (\$ 22,1)

Ce court extrait historique permet d'infirmer l'hypothèse de Daniel J. Taylor qui, à partir de la présence des mêmes proportions continues et discontinues dans le $D e$ lingua Latina et dans le passage sur la justice distributive de l'Éthique à Nicomaque d'Aristote (1131a29-1131b9), affirme : «I think it altogether likely that Varro had read and studied this section of Aristotle's Nicomachean Ethics and that the Greek text inspired and directly influenced him to formulate abstract mathematical models of inflection » (1996a, p. 148). Sans considérer les difficultés qui tiennent à l'hypothèse indémontrable d'une connaissance directe d'Aristote par Varron, il nous semble généralement plus opportun de privilégier l'idée selon laquelle un traité de mathématiques grec aurait transmis à Varron des connaissances parfaitement triviales à l'époque et exprimées dans un langage technique figé, dont les échos sont encore sensibles deux siècles plus tard, en milieu néo-pythagoricien.

Après avoir expliqué son modèle, Varron introduit l'opposition entre, d'une part, les proportions discontinues dont des formes casuelles constituent les quatre termes $(\S 47)$ :

$$
r e x_{\text {nom. }}: r e g i_{\text {dat. }}[\text { roi }]:: \operatorname{lex} x_{\text {nom. }}: \operatorname{leg} i_{\text {dat. }}[\text { loi }]
$$

32 Cf. notamment Roscher 1906, p. 109-142.

$33 C f$. par exemple, dans le célèbre passage arithmologique du De opificio mundi de Philon, les $\S 108-110$

$34 C f$. Varron hebd. frg. apud Aulu-Gelle 3,10,14. Pour la théorie des « jours critiques », $c f$. les sources rassemblées par Roscher (1906, p. 60-86), qui remarque que le quatrième jour est pris en compte par la tradition authentiquement hippocratique, alors qu'il ne figure pas dans les traités Sur les épidémies du corpus hippocratique.

35 Cf. notamment Favonius Eulogius 14,3, p. 20,6-9 Scarpa: musici uero septem uocum discrimina duobus tetracordis pro rata portione modulatis efficiunt, una corda communi, quae utriusque concentum harmoniae modificatione componat «Les musiciens en vérité établissent sept intervalles musicaux avec deux tétracordes agencés selon une proportion bien déterminée et dont une corde soit en commun, qui puisse produire leur accord réciproque par une modification de la hauteur du son ».

36 Dans son Exposition des connaissances utiles à la lecture de Platon, Théon de Smyrne emploie le même type d'opposition conceptuelle, même si son vocabulaire est un peu différent : sunekhés vs. diēirēménē analogía (p. 82,10 Hiller). D'autres auteurs présentent de brefs excursus historiques comparables à celui de Nicomaque : $c f$. la synthèse de Vitrac 1994, p. 497-501. 
et d'autre part, les proportions continues à trois termes, où figurent des temps verbaux relevant soit de l'infectum soit du perfectum ${ }^{37}$ :

$$
\text { legebam }_{\text {impt. }}: \operatorname{lego}_{\text {prés. }}:: \text { lego }_{\text {prés. }}: \text { legam }_{\text {fut. }} \text { [lire] }
$$

Enfin, il remarque que toutes les proportions peuvent être ramenées au schéma quadripartite ( $\$ 49$ quadripertita [...] forma $)^{38}$, même si -comme dans le cas des temps verbaux - elles donnent l'impression d'avoir un nombre inférieur de membres, ou bien d'en avoir davantage (\$ 49-50a). Pour démontrer ce dernier cas de figure, Varron semble faire appel à l'idée selon laquelle l'identité de rapport demeure stable dans la somme des antécédents de proportions différentes avec les mêmes conséquents, comme l'avait démontré Euclide dans le théorème 24 du livre $\mathrm{V}$ de ses Éléments ${ }^{39}$ :

$$
\text { A : B :: C : D et } \mathrm{E}: \mathrm{B}:: \mathrm{F}: \mathrm{D} \text { impliquent }(\mathrm{A}+\mathrm{E}): \mathrm{B}::(\mathrm{C}+\mathrm{F}): \mathrm{D}
$$

L'exemple numérique du De lingua Latina n'est peut-être pas très bien choisi ${ }^{40}$, mais ce type de démonstration permet d'expliquer :

1) les hétéroclisies, où deux formes du même cas oblique sont rattachées au même nominatif; par exemple, avec les noms propres «Diomède » et $\ll$ Hercule $»^{41}$ :

$$
\left[\text { Diomedi }_{\text {gén. }}+\text { Diomedis }_{\text {gén. }}\right]: \text { Diomedes }_{\text {nom. }}::\left[\text { Herculi }_{\text {gén. }}+\text { Herculis }_{\text {gén. }}\right]: \text { Hercules }_{\text {nom. }}
$$

2) les syncrétismes, où le double nominatif d'un nom propre qui a un masculin

37 Varron insiste sur la nécessité d'établir des comparaisons entre les temps de l'infectum, comme il vient de le faire, ou entre les temps du perfectum, sans les mêler (§ 48). Comme le remarque Taylor (1996a, p. 27), « We must notice two things in particular. First, Varro correctly recognizes aspect in Latin, i.e., the distinction between infectum (progressive) and perfectum (perfective). Second, Varro also identifies the future perfective indicative as in tutudero and amatus ero, and he is the only ancient grammarian to do so ».

38 Conso (1990, p. 799) remarque que dans ce passage forma pourrait se traduire par « série de nombres disposés de façon à mettre en évidence le rapport proportionnel, disposition d'une proportion ».

39 Cf. Vitrac 1994, p. 123-125; Acerbi 2007, p. 341-342.

40 Cf. Varron ling. 10,49 quemadmodum ad tria unum et duo, sic ad sex duo et quattuor, quae tamen quadripertita comprehenditur forma, quod bina ad singula conferuntur « Un et deux sont à trois comme deux et quatre sont à six, ce qui peut être exprimé par une formule à quatre termes où l'on a la mise en rapport de couples avec de termes isolés ». Taylor (1977, p. 320-321) est trop critique à l'égard de cet exemple : «A third mathematical proportion is rather interesting if for no other reason than that it does not provide a correct model for the grammatical data simply because it is not possible to do so. [...] The linguistic analogia demands two words which bear an equivalent ratio to a third word but which are themselves not equivalent, and there can be no parallel for this in simple numerical relationships ». La quadripertita forma évoquée par Varron, ainsi que le mot bina, qui pourrait désigner un couple de nombres qui doivent être additionnés, nous paraissent corroborer notre hypothèse : il ne faut certainement pas suivre à la lettre cette comparaison et s'attendre à ce que Varron «additionne» deux nominatifs ou deux génitifs; il suffit que ceux-ci soient considérés comme " associés ». Une interprétation a minima consisterait simplement à dire que deux ou plusieurs couples de nombres peuvent être insérés dans des proportions où l'autre couple demeure stable (par ex. $1: 3:: 2: 6$ et $2: 3:: 4: 6$ ).

41 L'hétéroclisie du nom Hercules est un problème recurrent pour les analogistes : $c f$. déjà Varron ling. 8,26. 
et un féminin se fléchit en une seule forme de cas oblique ; par exemple, avec les noms de deux gentes romaines ${ }^{42}$ :

$\left[\right.$ Baebiei $_{\text {nom. m. }}+$ Baebiae $\left._{\text {nom. f. }}\right]:$ Baebieis $_{\text {dat./abl. }}::\left[\right.$ Caelii $_{\text {nom. m. }}+$ Caeliae $\left._{\text {nom. . . }}\right]:$ Caeliis $_{\text {dat./abl. }}$

\section{FORMVLA : POSTÉRITÉ DU MOT ET CONCLUSION}

La série de parallèles que nous avons évoqués permet de mesurer l'apport des mathématiques à la réflexion varronienne sur la part d'analogie qui opère dans le système linguistique latin. Si Varron avait commencé à esquisser une ébauche de paradigme flexionnel dans l'image de la tabula lusoria, ce n'est qu'à l'aide des proportions qu'il parvint à montrer les processus morphologiques réguliers, rentrant dans des grilles bidimensionnelles. Dans cette démarche il dut être influencé par la lecture d'œuvres grecques, où figuraient des diagrámmata numériques.

La constitution des paradigmes flexionnels, que Varron appelle formulae même en dehors de leur représentation graphique, représente ainsi un des résultats les plus intéressants de l'interaction entre la grammaire et les modèles mathématiques ${ }^{43}$. Qu'il s'agisse de la création d'un vrai objet théorique, doté d'un nom technique spécifique, cela ressort de deux autres occurrences du terme formula. Au livre IX du De lingua Latina, quand il s'occupe des lacunes dans la conjugaison verbale, Varron résume ses arguments en renvoyant à ce qu'il avait déjà dit sur la déclinaison nominale (§ 75-79) :

S'il arrive dans ce domaine [sc. celui de la flexion verbale] ce qui se produit dans l'autre [ $s c$. celui de la flexion nominale], à savoir que, dans le paradigme, le point de départ manque ou qu'il soit d'un autre type, suivant la même proportionnalité de rapports qui existe dans l'autre domaine, nous disons pourquoi l'analogie est néanmoins préservée. De même, si, comme dans le domaine des noms, la flexion verbale a un point de départ et si la transition aux formes fléchies se produit selon un des paradigmes, une fois ce paradigme pris en compte, on peut voir plus aisément d'où les autres formes verbales sont fléchies ${ }^{44}$.

42 Ce dernier exemple permet d'expliquer la remarque finale de Varron : « À partir de deux nominatifs semblables on peut avoir des formes déclinées semblables, comme il arrive dans nemus [bois, s.] : olus [légume, s.] :: nemora [pl.] : olera [pl.] ; d'autres formes semblables peuvent être déclinées à partir de nominatifs différents, comme dans les pronoms, hunc, istunc [acc.] de hic, iste [nom.] » (§50b).

43 Ce fait est reconnu de manière générale par les interprètes de Varron. $C f$. par exemple Taylor (1974, p. 48-49) : «By positing complete paradigmatic identity as the basis of comparison and classification, the morphophonemic patterns were as rigid as exact as numerical proportions »; (1977, p. 322-323) : «Descriptively the conclusion is clear - words, in order to be classified together, must exhibit, in terms of their phonological form and morphological content, the same type of exact relationship as numbers in mathematical proportions. [...] By basing linguistic classification on complete paradigmatic identity, Varro was arguing for morphophonemic patterns, that is, embryonic declensions and conjugations, which were as precise and as exact as numerical proportions ».

$44 C f$. Varron ling. 9,103 quare ut illic fit, si [c] hic item acciderit in formula, ut aut caput non sit aut ex alieno genere sit, proportione eadem quae illic dicimus $\{$ qua ... diximus $\mathrm{L}$. Spengel, Madvig, diximus Mette\}, cur ni[c]hilominus seruetur analogia; item, si[c] ut illic caput suum habebit et in obliquis casibus transitio erit in aliquam formulam, qua assumpta reliqua facilius possint uideri uerba, unde sint declinata. 
Enfin, au livre $\mathrm{X}$, il renvoie à une section non conservée de son œuvre, où il traite de formulis uerborum (ling. 10,33), à savoir des conjugaisons verbales ${ }^{45}$.

Cet emploi spécialisé de formula, ainsi que la représentation graphique que ce mot sous-tend, peuvent être considérés comme un des legs « cachés » de la tradition varronienne aux manuels de grammaire scolaire de l'antiquité tardive. Nous résumons synthétiquement les données essentielles du dossier :

1) Charisius (167,12-14 Barwick), dans la section De analogia tirée de Julius Romanus, à propos des hellénismes neutres en -mata, remarque qu'ils ont les génitif et datif du pluriel relevant de deux schémas flexionnels distincts $(e t$ ideo haec et eius modi ex alia formula genetiuum pluralem et ex alia datiuum sumunt) et, par l'intermédiaire de Pline (Dubius sermo frg. 82 Della Casa), cite Varron (De similitudine uerborum frg. 52 Goetz \& Schoell ${ }^{46}$, qui voudrait attribuer la terminaison en -bus aux datif et ablatif du pluriel de ces noms.

2) Diomède ( $G L$ 1,347,23-28) énumère les verbes de la «troisième » conjugaison, qui ont un - $i$ - bref ou long devant la désinence $-s$ de la deuxième personne du singulier : ainsi lego, leğ $\breve{l}-s$ et audio, audī-s. Leur futur se termine en - am à la première personne, sauf pour les verbes qui suivent le schéma flexionnel de la «deuxième » conjugaison (type moneo, moness) juste à la première personne, avec un - $e$ - bref, et qui ont un futur en - $b o$ (par exemple eo is ibo, queo quis quibo): sed etiam in bo, in his tamen dumtaxat quae, cum producta sint, secundae coniugationis formulam in prima persona seruant. $\mathrm{La}$ même remarque concerne la formation des parfaits en -ui de cette classe de verbes (371,4-8 cum sint tertiae coniugationis, secundae formulam seruant in persona prima dumtaxat).

3) Phocas, dans son De nomine et uerbo, remarque que, indépendamment de leur genre, les monosyllabes suivent la flexion des noms de la troisième déclinaison (31,12-14 Casaceli : monosyllaba congesta praeposui, quae diuersi quidem sunt generis, tertii autem ordinis formulam tribus exceptis secuntur) ; considère comme une exception les mots cardo cardinis et ordo ordinis, qui tout en présentant un $-d$ - avant la terminaison -o, comme hirundo hirundinis, f. " hirondelle», sont masculins et, au lieu de suivre dans leur flexion le modèle des masculins qui ont le même nominatif mais qui n'ont pas d'apophonie aux cas obliques (type praedo praedonis), se conforment aux féminins (33,23-24 C. hic cardo, hic ordo, quae superiorem formulam sequuntur); assigne le schéma flexionnel de la troisième déclinaison aux neutres en -ar (36,3-4 C. haec quoque tertiae declinationis formulam secuntur); affecte les masculins en -us qui sont des noms propres ou qui forment le féminin au schéma flexionnel de la deuxième déclinaison (41,21-23 C. illa masculina secundae declinationis accipiunt formulam, quae aut propria sunt aut in femininum genus transeunt, ut hic Tullius, clarus (facit enim clara).

4) dans les Regulae attribuées à Saint Augustin, l'expression ad hanc formulam declinare est très fréquente $(G L 5,499,14.23 ; 501,35-36 ; 502,3-4.9)$. Cela

45 Cf. Goetz \& Schoell 1910, p. 295 comm. ad 201,20 sqq.

$46 C f$. également l'extrait du De utilitate sermonis frg. 53 Goetz \& Schoell, chez Charisius 157,3-9 Barwick. 
s'explique par la nature elle-même du texte, qui n'est qu'un recueil de mots fléchis, dont certains servent de paradigme à d'autres. En GL 5,499,19-25 l'auteur prend en compte les neutres en -ur du type murmur, murmora qui ont les cas obliques en -or- et remarque : ad hanc formulam similia declinabis, ut ebur ebora, femur femora, sulphur sulphora, fulgur fulgora. sed ex hac formula non multa inueniuntur.

Les grammatici Latini tardifs ont certainement trouvé dans les formulae un moyen pratique pour représenter les phénomènes que Varron, en premier, s'efforçait d'organiser en un tout cohérent, fondé sur des processus de flexion réguliers. $\mathrm{Si}$ on assiste ainsi à un cas de «cryptotradition », où la transmission d'outils théoriques doit être imputée moins à la circulation des textes qu'à celle de la pensée d'un auteur ${ }^{47}$, les discontinuités et les ruptures avec le modèle ne sauraient néanmoins être sous-estimées : au lieu de mettre en lumière le caractère productif, voire dynamique, de la morphologie, en dérivant à l'instar de Varron des grilles bidimensionnelles de l'intersection entre proportions arithmétiques, les artigraphes se limiteront à établir des taxinomies figées, dont le caractère statique révèle leur démarche normative.

Adresse de l'auteur : <agarc@libero.it>

\section{BIBLIOGRAPHIE}

\section{Sources primaires}

Acerbi, Fabio (2007). Euclide. Tutte le Opere, Milano, Bompiani.

Arnaldez, Roger (1961). Philon d'Alexandrie. De opificio mundi, Paris, Éditions du Cerf.

Barwick, Karl (1964). Flavii Sosipatri Charisii artis grammaticae libri V, Editio stereotypa correctior editionis prioris. Addenda et corrigenda collegit et adiecit F. Kühnert, Lipsiae, Teubner.

Bertier, Jeanine (1978). Nicomaque de Gérase. Introduction arithmétique, Paris, Vrin.

Casaceli Francesco (1974), Foca. De nomine et uerbo, Napoli, Libreria scientifica editrice.

D’Ooge, Martin L., Robbins, Frank E. \& Karpinski, Louis Ch. (1926). Nicomachus of Gerasa. Introduction to Arithmetic, New York - London, MacMillan.

Dupont-Roc, Roseline \& Lallot, Jean (1981). Aristote. La poétique, Paris, Seuil.

Edelstein, Ludwig \& Kidd Ian G. (1988-1999). Posidonius, Cambridge, Cambridge University Press, 4 vol.

Edelstein, Ludwig \& Kidd, Ian Gray (1988). Posidonius, 2, The Commentary, Cambridge, University Press.

Goetz, Georg \& Schoell, Friedrich (1910). M. Terenti Varronis de lingua Latina quae supersunt, Lipsiae, Teubner.

Guillaumin, Jean-Yves (1995). Boèce. Institution arithmétique, Paris, Les Belles Lettres.

Hiller Eduard (1878). Theoni Smyrnaei philosophi platonici Expositio rerum mathematicarum ad legendum Platonem utilium, Lipsiae, Teubner.

Hoche, Richard (1866). Nicomachi Geraseni Pythagorei Introductionis Arithmetica libri II, Lipsiae, Teubner.

Keil, Heinrich (1855-1880). Grammatici Latini, Lipsiae, Teubner: vol. 1. Flavii Sosipatri Charisii artis grammaticae libri V. Diomedis artis grammaticae libri III. Ex Charisii

47 Pour ce modèle historiographique en linguistique, $c f$. Giannini 1996, p. 195-197. 
arte grammatica excerpta, vol. 2. Prisciani institutionum grammaticarum libri IXII ex recensione Martini Hertzii, vol. 3. Prisciani institutionum grammaticarum libri XIII-XVIII ex recensione Martini Hertzii. Prisciani opera minora ex recensione Henrici Keilii, vol. 4. Probi Donati Seruii qui feruntur de arte grammatica libri ex recensione Henrici Keilii. Notarum laterculi ex recensione Theodori Mommseni, vol. 5. Artium scriptores minores. Cledonius, Pompeius, Iulianus excerpta ex commentariis in Donatum. Consentius, Phocas, Eutyches, Augustinus, Palaemon, Asper. De nomine et pronomine. De dubiis nominibus. Macrobii excerpta, vol. 6. Scriptores artis metricae: Marius Victorinus, Maximus Victorinus, Caesius Bassus, Atilius Fortunatianus, Terentianus Maurus, Marius Plotius Sacerdos, Rufinus, Mallius Theodorus, Fragmenta et excerpta metrica, vol. 7. Scriptores de orthographia: Terentius Scaurus, Velius Longus, Caper, Agroecius, Cassiodorius, Martyrius, Beda, Albinus. Audacis excerpta, Dosithei Ars grammatica, Arusiani Messii Exempla elocutionum, Cornelii Frontonis liber de differentiis, Fragmenta grammatica.

Kumaniecki, Kazimierz F. (1969). M. Tulli Ciceronis scripta quae manserunt omnia, Fasc. 3, De oratore, Lipsiae, Teubner.

Marshall, Peter K. (1968). Auli Gellii noctes Atticae, Oxford, Oxford University Press, 2 vol.

Mette, Hans Joachim (1952). Parateresis : Untersuchungen zur Sprachtheorie des Krates von Pergamon, Halle (Saale), Niemeyer.

Scarpa, Luigi (1974). Favonii Eulogii disputatio de somnio Scipionis, Padova, Accademia patavina di scienze, lettere ed arti, Università di Padova, Istituto di filologia latina.

Spengel, Leonard \& Andreas (1885). M. Terenti Varronis de lingua Latina libri, Berolini, Weidmann.

Taylor, Daniel J. (1996a). Varro. De lingua Latina X, Amsterdam - Philadelphia, Benjamins.

Theiler, Willy (1982). Poseidonius, Die Fragmente. Berlin - New York, De Gruyter, 2 vol.

Vitrac, Bernard (1994). Euclide. Les Éléments, 2, Livres V-VI, Paris, Presses Universitaires de France.

Ziegler, Konrat (1969). M. Tulli Ciceronis scripta quae manserunt omnia, Fasc. 39, De re publica, Lipsiae, Teubner.

\section{Sources secondaires}

Barresi, Paolo (1998-2000). «Su una tabula lusoria da Gortina », Annuario della Scuola Archeologica di Atene 60-62, 249-271.

Bean, George Ewart (1968). Turkey's Southern Shore, London, E. Benn.

Callanan, Christopher K. (1987). Die Sprachbeschreibung bei Aristophanes von Byzanz, Göttingen, Vandenhoeck und Ruprecht.

Collart, Jean (1954). Varron grammairien latin, Paris, Les Belles Lettres.

Conso, Danièle (1990). Forma: étude sémantique, Thèse d'État, Université de Paris 4 - Sorbonne.

Ducos, Michèle (1994), art. « Diodotos n. 134 », DPhA 2, 796.

Duso, Antonella (2006). « L'analogia in Varrone », Oniga, Renato \& Zennaro, Luigi (éds.), Atti della Giornata di Linguistica Latina, Venezia, Università Ca’ Foscari, 9-20.

Erbse, Hartmut (1980). «Zur normativen Grammatik der Alexandriner », Glotta 58, 236258.

Fellmann, Rudolf (1983). « Abacus », Antike Welt 14.1, 36-40.

Garcea, Alessandro (2007). «César et les paramètres de l'analogie », Basset, Louis, Biville, Frédérique, Colombat, Bernard, Swiggers, Pierre \& Wouters, Alfons (éds.), Bilinguisme et terminologie grammaticale gréco-latine, Leuven - Paris - Dudley (Mass.), Peeters, 339-357.

Giannini, Stefania (1996). Percorsi metalinguistici : Giuliano di Toledo e la teoria della grammatica, Milano, Franco Angeli.

Grilli, Alberto (1979). « Sul numero sette», Scritti in onore di Benedetto Riposati. Studi 
su Varrone sulla retorica storiografia e poesia latina, vol. 1, Rieti, Centro di Studi Varroniani - Milano, Università del Sacro Cuore, 203-219.

Ju, Anna E. (2006). « Posidonius on Incorporeal Limits », Journal of Greco-Roman Studies $25,329-358$.

Ju, Anna E. (à paraître). «Posidonius on "Old Authorities" and Pythagoreanism », conference paper (Cambridge, Faculty of Classics, October 2007).

Law, Vivien (2003). The History of Linguistics in Europe from Plato to 1600, Cambridge, University Press.

Moatti, Claudia (1997). La raison de Rome. La naissance de l'esprit critique à la fin de la République (II -I $^{\text {er }}$ siècle avant Jésus-Christ), Paris, Seuil.

Piras, Giorgio (1998). Varrone e i poetica uerba. Studio sul settimo libro del de lingua Latina, Bologna, Pàtron.

Pohlenz, Max (1918). M. Tulli Ciceronis scripta quae manserunt omnia, Fasc. 44, Tusculanae disputationes, Lipsiae, Teubner.

Roscher, Wilhelm H. (1906). Die Hebdomadenlehren der griechischen Philosophen und $\ddot{A r z t e}$, Leipzig, Teubner.

Schironi, Francesca (2007). «Analogia, proportio, ratio: Loan Words, Calques, and Reinterpretations of a Greek Technical Word», Basset, Louis, Biville, Frédérique, Colombat, Bernard, Swiggers, Pierre \& Wouters, Alfons (éds.), Bilinguisme et terminologie grammaticale gréco-latine, Leuven, Paris - Dudley (Mass.), Peeters, 321338.

Siebenborn, Elmar (1976). Die Lehre von der Sprachrichtigkeit und ihren Kriterien. Studien zur antiken normativen Grammatik, Amsterdam, Grüner.

Swiggers, Pierre (1997). Histoire de la pensée linguistique, Paris, Presses Universitaires de France.

Taylor, Daniel J. (1974). Declinatio : a Study of the Linguistic Theory of Marcus Terentius Varro, Amsterdam, Benjamins.

Taylor, Daniel J. (1977). « Varro's Mathematical Models of Inflection », Transactions of the American Philological Association 107, 313-323.

Taylor, Daniel J. (1978). «Ordo in Book X of Varro's De Lingua Latina », Collart, Jean (éd.), Varron. Grammaire antique et stylistique latine, Paris, Les Belles Lettres, 71-74.

Taylor, Daniel J. (1996b). « Style and Structure in Varro ? », Swiggers, Pierre \& Wouters, Alfons (éds.), Ancient Grammar : Context and Content, Leuven - Paris, Peeters, 91103. 